\title{
Analysis of the left atrial function using two- dimensional strain in patients with recent diagnosis of hereditary hemochromatosis
}

\section{Ariel Karim Saad ( $\sim$ arielsaad@gmail.com )}

Hospital de Clínicas José de San Martín: Hospital de Clinicas Jose de San Martin https://orcid.org/0000-0002-6495-997X

José Martín Aladio Hospital de Clínicas José de San Martín: Hospital de Clinicas Jose de San Martin

Florencia Yamasato

Hospital de Clínicas José de San Martín: Hospital de Clinicas Jose de San Martin

\section{Veronica Ines Volberg}

Hospital de Clínicas José de San Martín: Hospital de Clinicas Jose de San Martin

\section{Esteban Gonzalez Ballerga}

Hospital de Clínicas José de San Martín: Hospital de Clinicas Jose de San Martin

Juan Antonio Sorda

Hospital de Clínicas José de San Martín: Hospital de Clinicas Jose de San Martin

Jorge Daruich

Hospital de Clínicas José de San Martín: Hospital de Clinicas Jose de San Martin

Ricardo Alfredo Perez de la $\mathrm{Hoz}$

Hospital de Clínicas José de San Martín: Hospital de Clinicas Jose de San Martin

\section{Original Article}

Keywords: Hereditary Hemochromatosis, speckle-tracking echocardiography, left atrial function, atrial strain

Posted Date: February 18th, 2021

DOI: https://doi.org/10.21203/rs.3.rs-201689/v1

License: (c) (i) This work is licensed under a Creative Commons Attribution 4.0 International License. Read Full License

Version of Record: A version of this preprint was published at Current Problems in Cardiology on May 1st, 2021. See the published version at https://doi.org/10.1016/j.cpcardiol.2021.100903. 


\section{Abstract}

\section{Background}

Hereditary Hemochromatosis $(\mathrm{HH})$ is a genetic condition associated with a systemic iron overload. Heart failure is an important cause of mortality. It has been demonstrated early stages of systolic and diastolic left ventricular dysfunction in previous studies. The aim of the study is to compare the left atrial (LA) function between asymptomatic $\mathrm{HH}$ patients and a control group using 2D speckle tracking.

\section{Methods}

Prospective study. LA strain, LA strain rate and LA volumetric parameters during the reservoir, conduit and contraction phases were studied. The LA Stiffness Index was calculated by the ratio between E/e and LA reservoir strain.

Results

30 patients with $\mathrm{HH}$ ( $90 \%$ males, $47 \pm 18$ years old) and 30 healthy controls ( $85 \%$ males, $45 \pm 13$ years old) were included. LA volume was similar in both groups. No differences were observed in LA ejection fraction (EF), LA passive EF and LA active ejection fraction between both groups. On the contrary, the $\mathrm{HH}$ group had lower LA strain during the reservoir ( $31.5 \pm 6.5 \%$ vs $38.3 \pm 7.9 \% ; p=0.002)$, and conduit phases $(-18 \pm 7 \%$ vs $-23.3 \pm 6.4 \% ; p=0.01)$ and lower LA conduit strain rate $\left(-1.7 \pm 0.7 \mathrm{seg}^{-1}\right.$ vs $-2.1 \pm 0.5 \mathrm{seg}^{-1} ; p$ $=0.02)$ than controls. The LA stiffness index was significantly higher in the $\mathrm{HH}$ group $(0.25 \pm 0.9$ vs 0.19 $\pm 0.6 ; p=0.01$ ).

Conclusions

Early abnormalities in the LA function could be detected by using 2D speckle tracking study despite no evidence of changes in atrial size or volumetric parameters.

\section{Introduction}

Hereditary hemochromatosis $(\mathrm{HH})$ is a genetic disorder in which there is a systemic iron $(\mathrm{Fe})$ overload, mainly caused by a deficit in the production of hepcidin or the activity of the hepcidin-ferroportin complex. Hepcidin deficiency increases Fe release from splenic macrophages and cells of the small intestine into plasma. The increase in the plasma levels of Fe causes its accumulation in parenchymal cells of different organs, mainly liver, pancreas and heart (1). The clinical manifestations of heart compromise are usually seen at late stages of the disease, and it is described that $\mathrm{HH}$ can cause dilated or restrictive cardiomyopathy (2). However, previous studies carried out in the early stages of HH have shown incipient alterations in both systolic and diastolic function. The more sensitive the techniques used to evaluate alterations in myocardial function, the more possibilities there are to detect them $(3,4)$. 
The main function of the left atrium is to modulate ventricular filling through the reservoir, conduit, and pump phases. The study of atrial function allows a better understanding of cardiac diastole (5). Its analysis has shown usefulness as a prognostic marker in patients with heart failure and preserved ejection fraction (EF), valvular heart disease and atrial fibrillation (6). With the new imaging techniques, such as the analysis of the deformation by means of speckle tracking, it is possible to analyze in a sequential way the different periods of the atrial cycle with high feasibility, allowing to quantify its functioning and consequently, to be able to anticipate initial alterations of the function even before the increase in size, which could promote earlier treatment of the underlying disease.

The objective of this study is to compare the left atrial (LA) function by two-dimensional strain between asymptomatic, treatment-naive patients with a recent diagnosis of $\mathrm{HH}$ and a control group.

\section{Methods}

\section{Study Population}

We included asymptomatic patients with a recent diagnosis of $\mathrm{HH}$ according to the following criteria: serum ferritin $>200 \mathrm{ng} / \mathrm{ml}$ in women or $>300 \mathrm{ng} / \mathrm{ml}$ in men and/or transferrin saturation $>45 \%$ without a secondary cause that justifies the Fe overload and liver biopsy compatible with $\mathrm{HH}$ with mild or no fibrosis $(7,8)$. The patients had not received any type of specific treatment and agreed to participate in the study by signing the corresponding consent. Recruitment was carried out in the period between November 2017 and May 2019 in the hepatology offices of the Gastroenterology Division of the Hospital de Clinicas: "José de San Martin" (University of Buenos Aires, Argentina). We excluded those patients with structural heart disease of any etiology (ischemic, hypertrophic, valvular, idiopathic, etc.), as well as those with a history of atrial fibrillation, diabetes mellitus, left bundle branch block, and poor acoustic window. The results were compared with a control group taking into account sex, age and risk factors for cardiovascular disease, selected from individuals who requested a routine evaluation in the Cardiology Division office of the same hospital. These patients had a negative physical stress test for myocardial ischemia, carried out by ergometry or stress echocardiography.

\section{Echocardiography}

Echocardiographic evaluation was performed in the echocardiography laboratory with a Vivid E9 or E95 (GE Health Care, Milkwaukee, USA) equipment, with M5S multi-frequency transducers. The images were acquired with a frame rate between $60-80 \mathrm{frames} / \mathrm{sec}$ at rest. The evaluation of cardiac chamber size, as well as transvalvular flows, was performed according to the guidelines of the American Society of Echocardiography (9). In addition to conventional echocardiographic evaluation, longitudinal strain and strain rate of the left atrium were analyzed in the apical four-chamber view. This was carried out offline, with the EchoPAC PC v205 software provided by the same manufacturer, semi-automatically, that is, marking the region of interest in the atrial wall and making the necessary adjustments to achieve adequate monitoring of the acoustic markers (speckles). Based on current recommendations, the values of the curves of the six segments were averaged during the LA reservoir, conduit and pump phases. The 
quality of the speckles follow-up was validated both by the program and by the operator, having the possibility of excluding from the analysis those segments with poor quality tracking (Fig. 1). The LA strain during reservoir phase (LASr) was estimated as the difference of the strain value at mitral valve opening minus ventricular end-diastole, the LA strain during conduit phase (LAScd) was estimated as the difference of the strain value at the onset of atrial contraction minus mitral valve opening, and the LA strain during contraction phase (LASct) with the difference of the strain value at the ventricular enddiastole minus onset of atrial contraction. The strain rate values of the different phases were estimated with their peak velocity value (6). The estimated volumetric parameters were: LA ejection fraction (LAef) as the percentage between maximum and minimum volume, passive ejection fraction (LApef) as the percentage between maximum volume and pre-atrial contraction volume, and the active ejection fraction (LAaef) as the percentage between the pre-contraction volume and the minimum volume (10). The stiffness index (SI) was estimated as the ratio of E/e' to $\operatorname{LASr}(11,12)$.

\section{Additional evaluations of patients with $\mathrm{HH}$}

In addition to the echocardiographic evaluation and liver biopsy, patients with $\mathrm{HH}$ underwent a genetic examination, with the most common mutations of the disease in our region (C282Y, H63D and S65C) and a magnetic resonance imaging (MRI) to evaluate the liver and heart Fe overload using the T2* sequence.

\section{Statistical analysis}

The analysis was performed with the statistical package IBM SPSS $®$ Statistics v20 program. Nominal variables were expressed as percentages in relation to the total number of cases and quantitative variables as mean and standard deviation or median and interquartile range, as appropriate. The normality of the distributions was analyzed with the Kolmogorov-Smirnov or Shapiro-Wilk test. For statistical comparison between groups, the corresponding hypothesis tests (Student's t, Mann-Whitney, chi square) were used according to the type of variable and the distribution of the data. Intra-observer and interobserver variability were analyzed using the intraclass correlation coefficient. A significance threshold of 0.05 was used.

\section{Ethical considerations}

The study was approved by the Bioethics Committee of the hospital and was carried out in accordance with current regulations for observational studies, respecting the principles of the Declaration of Helsinki.

\section{Results}

\section{Study Population}

A total of 30 patients with $\mathrm{HH}$ (mean age $47 \pm 18$ years) and 30 controls (mean age $45 \pm 13$ years) were included in the study. No significant differences were observed between the groups in terms of heart rate, blood pressure, and profile of cardiovascular risk factors. In Table 1 these values are detailed, as well as the plasma parameters of iron metabolism of patients with $\mathrm{HH}$. 
Table 1

Population characteristics and metabolic profile of patients with $\mathrm{HH}$.

\begin{tabular}{|llll|}
\hline & HH $(\mathbf{n}=30)$ & Control $(\mathbf{n = 3 0})$ & $\mathbf{p}$ \\
\hline Age (years) & $47 \pm 18$ & $45 \pm 13$ & 0,54 \\
\hline Weight $(\mathrm{kg})$ & $80 \pm 15$ & $72 \pm 11$ & 0,02 \\
\hline BMI $\left(\mathrm{kg} / \mathrm{m}^{2}\right)$ & $25,6 \pm 6,1$ & $25 \pm 2,6$ & 0,75 \\
\hline Heart rate & $68 \pm 10$ & $70 \pm 9$ & 0,75 \\
\hline SBP $(\mathrm{mmHg})$ & $131 \pm 13$ & $129 \pm 13$ & 0,59 \\
\hline DBP $(\mathrm{mmHg})$ & $80 \pm 7$ & $80 \pm 7$ & 0,99 \\
\hline Hypertension & $27 \%$ & $25 \%$ & 0,45 \\
\hline Hyperlipidemia & $14 \%$ & $21 \%$ & 0,65 \\
\hline Tobacco use & $13 \%$ & $14 \%$ & \\
\hline Serum ferritin level $(\mathrm{ng} / \mathrm{mL})$ & $882 \pm 574$ & & \\
\hline Serum iron level $(\mu \mathrm{g} / \mathrm{mL})$ & $149 \pm 42$ & & \\
\hline Serum transferrin level $(\mu \mathrm{gg} / \mathrm{mL})$ & $229 \pm 33$ & & \\
\hline Transferrin saturation $(\%)$ & $46 \pm 15$ & & \\
\hline TIBC ( $\mu$ g $/ \mathrm{mL})$ & $310 \pm 41$ & & \\
\hline Hematocrit $(\%)$ & $42 \pm 2,7$ & & \\
\hline DBP: diastolic blood $\mathrm{pressure.} \mathrm{SBP:} \mathrm{systolic} \mathrm{blood} \mathrm{pressure;} \mathrm{TIBC:} \mathrm{Total} \mathrm{Iron-Binding} \mathrm{Capacity}$ & \\
\hline
\end{tabular}

Regarding the genetic analysis of patients with $\mathrm{HH}$ (HFE gene), the two most frequent mutations were $\mathrm{C} 282 \mathrm{Y}$ and H63D. One patient (4\%) was homozygous for C282Y, 3 patients were homozygous for the H63D mutation (11\%), 3 patients were heterozygous for the $\mathrm{C} 282 \mathrm{Y}$ mutation (11\%), 5 patients were heterozygous for H63D (21\%) and in the rest of the patients (53\%) such mutations were not detected. All patients with $\mathrm{HH}$ had moderate or severe liver iron overload estimated by MRI. But no patient had Fe in the myocardium, with a T2* sequence greater than $20 \mathrm{msec}$ in 100\% of the cases (MED $25.5 \mathrm{msec}$ IQR 25-75 21-40 msec). Regarding the liver biopsy, $48 \%$ did not have fibrosis, and $52 \%$ had it in a mild stage.

\section{Conventional Echocardiography Data}

From the structural point of view, no significant differences were observed in terms of cardiac chambers size between the two groups. Although there is evidence of a trend towards a greater area of the right atrium (17 $\pm 6.5 \mathrm{~cm}^{2}$ vs $\left.15 \pm 3.4 \mathrm{~cm}^{2} ; \mathrm{p}=0.09\right)$ in individuals with $\mathrm{HH}$. Regarding systolic function, a lower 
EF was observed in patients with $\mathrm{HH}(59 \pm 4 \%$ vs $62 \pm 4 \% ; \mathrm{p}=0.01)$. With respect of diastolic function, although no differences were observed between the velocities of the $E$ and $A$ waves of both ventricles, the patients in the $\mathrm{HH}$ group had a lower mitral $\mathrm{E} / \mathrm{A}$ ratio $(1.18 \pm 0.5$ vs $1,45 \pm 0.5 ; \mathrm{p}=0.04)$ and more decreased septal tissue e' wave $(0.09 \pm 0.03 \mathrm{~m} / \mathrm{sec}$ vs $0.11 \pm 0.03 \mathrm{~m} / \mathrm{sec} ; \mathrm{p}=0.03)$. Table 2 summarizes the most relevant findings.

Table 2

Results of the evaluation with two-dimensional echocardiography and Doppler.

\begin{tabular}{|c|c|c|c|}
\hline & $H H(n=30)$ & Control $(n=30)$ & $\mathbf{P}$ \\
\hline LV diastolic diameter (mm) & $45,8 \pm 3,8$ & $45, \pm 4$ & 0,84 \\
\hline Septal thickness (mm) & $9,4 \pm 1,9$ & $9,1 \pm 1,1$ & 0,40 \\
\hline Posterior wall thickness (mm) & $8,8 \pm 1,2$ & $8,5 \pm 0,9$ & 0,36 \\
\hline LV mass $\left(\mathrm{g} / \mathrm{m}^{2}\right)$ & $74,6 \pm 20$ & $74,3 \pm 11$ & 0,94 \\
\hline LV EDV (mL) & $77 \pm 24$ & $75 \pm 2$ & 0,74 \\
\hline LV ESV (mL) & $31,5 \pm 10$ & $28,2 \pm 8,8$ & 0,20 \\
\hline LV EF (\%) & $59 \pm 4$ & $62 \pm 4$ & 0,01 \\
\hline LA area $\left(\mathrm{cm}^{2}\right)$ & $19,9 \pm 4,3$ & $18,5 \pm 2,1$ & 0,22 \\
\hline $\mathrm{RA}$ area $\left(\mathrm{cm}^{2}\right)$ & $17 \pm 3,5$ & $15,1 \pm 2,4$ & 0,09 \\
\hline Mitral E velocity (m/seg) & $0,72 \pm 0,16$ & $0,79 \pm 0,13$ & 0,05 \\
\hline Mitral A velocity (m/seg) & $0,67 \pm 0,26$ & $0,6 \pm 0,19$ & 0,20 \\
\hline Mitral E/A ratio & $1,18 \pm 0,5$ & $1,45 \pm 0,5$ & 0,04 \\
\hline Mitral E-velocity DT (mseg) & $191 \pm 41$ & $191 \pm 45$ & 0,99 \\
\hline TDI septal e' velocity (m/seg) & $0,09 \pm 0,03$ & $0,11 \pm 0,03$ & 0,03 \\
\hline Mitral E/e' ratio & $7,9 \pm 2$ & $7,5 \pm 1,8$ & 0,44 \\
\hline
\end{tabular}

\section{Echo 2D Strain Data}

In the analysis of LA mechanics, no differences were observed in volumes during the reservoir, conduit and pump phases, nor in volumetric ejection parameters. On the contrary, significant differences were detected at the strain level during the reservoir and conduit phases with a more depressed percentage in the $\mathrm{HH}$ group ( $31.5 \pm 6.5 \%$ vs $38.3 \pm 7.9 \% ; \mathrm{p}=0.002$ and $-18 \pm 7 \%$ vs $-23.3 \pm 6.4 \% ; p=0.01$ respectively), and a lower peak LASRc in HH patients $\left(-1.7 \pm 0.8 \mathrm{sec}^{-1} \mathrm{vs}-2.1 \pm 0.5 \mathrm{sec}^{-1} ; p=0.02\right)$. At the ventricular 
level, patients with $\mathrm{HH}$ had lower global early diastolic strain rate $\left(1.49 \pm 0.36 \mathrm{sec}^{-1}\right.$ vs $1.75 \pm 0.38 \mathrm{sec}^{-1}$; $p=0.009)$, and a significant correlation with reservoir strain was observed $(r=0.41 ; p=0.006)$ (Fig. 2$)$. The stiffness index was higher in patients with $\mathrm{HH}: 0.25 \pm 0.9$ vs $0.195 \pm 0.65 p=0.01$ (Table 3).

Table 3

Analysis of volumes and contractile mechanics of the left atrium using two-dimensional strain.

\begin{tabular}{|c|c|c|c|}
\hline & $\mathrm{HH}(\mathrm{n}=30)$ & Control $(n=30)$ & $\mathbf{P}$ \\
\hline LASr (\%) & $31,5 \pm 6,5$ & $38,3 \pm 7,9$ & 0,002 \\
\hline $\operatorname{LASRr}\left(\mathrm{seg}^{-1}\right)$ & $1,52 \pm 0,4$ & $1,67 \pm 0,35$ & 0,18 \\
\hline LAScd (\%) & $-18 \pm 7$ & $-23,3 \pm 6,4$ & 0,01 \\
\hline LASRcd $\left(\operatorname{seg}^{-1}\right)$ & $-1,7 \pm 0,8$ & $-2.1 \pm 0,5$ & 0,02 \\
\hline LASct (\%) & $-15 \pm 4,8$ & $-13,5 \pm 4$ & 0,23 \\
\hline LASRct $\left(\mathrm{seg}^{-1}\right)$ & $-1,8 \pm 0,52$ & $-1,9 \pm 0,47$ & 0,60 \\
\hline Maximal LA volume $\left(\mathrm{mL} / \mathrm{m}^{2}\right)$ & $28,2 \pm 12$ & $29,3 \pm 6,1$ & 0,68 \\
\hline Minimal LA volume $\left(\mathrm{mL} / \mathrm{m}^{2}\right)$ & $12,2 \pm 7$ & $11,7 \pm 2,8$ & 0,77 \\
\hline Pre-A volume $\left(\mathrm{mL} / \mathrm{m}^{2}\right)$ & $17,9 \pm 8$ & $18,7 \pm 4,2$ & 0,69 \\
\hline LAef (\%) & $57,5 \pm 10$ & $60 \pm 5$ & 0,32 \\
\hline LApef (\%) & $35 \pm 12$ & $36 \pm 9$ & 0,82 \\
\hline LAaef (\%) & $34 \pm 11$ & $36 \pm 9,9$ & 0,41 \\
\hline Stiffness index & $25,5 \pm 9,1$ & $19,5 \pm 6,4$ & 0,01 \\
\hline LV e'SR $\left(\mathrm{seg}^{-1}\right)$ & $1,49 \pm 0,36$ & $1,75 \pm 0,38$ & 0,009 \\
\hline \multicolumn{4}{|c|}{$\begin{array}{l}\text { LA: left atrial; LASr: strain during reservoir phase; LASRr: peak strain rate during reservoir phase; } \\
\text { LAScd: strain during the conduit phase; LASRcd: peak strain rate during conduit phase; LASct: strair } \\
\text { during contraction phase; LASRct: peak strain rate during contraction phase; LAef: ejectrion fraction } \\
\text { LApef: passive ejection fraction; LAaef: active ejection fraction; LV e'SR: left ventricular global early } \\
\text { diastolic strain rate. }\end{array}$} \\
\hline
\end{tabular}

No correlation was observed between the different types of deformation and the plasma parameters of Fe metabolism (Table 4). 
Table 4

Correlation between two-dimensional strain and iron parameters

\begin{tabular}{|c|c|c|c|c|c|c|}
\hline & \multicolumn{2}{|c|}{ Serum iron } & \multicolumn{2}{|c|}{ Serum ferritin } & \multicolumn{2}{|c|}{ Transferrin saturation } \\
\hline & $\mathrm{R}$ & $\mathrm{p}$ & $\mathrm{R}$ & $\mathrm{p}$ & $\mathrm{R}$ & $\mathrm{P}$ \\
\hline LASr (\%) & 0,20 & 0,42 & $-0,32$ & 0,18 & 0,37 & 0,12 \\
\hline LAScd (\%) & $-0,26$ & 0,28 & 0,47 & 0,04 & $-0,33$ & 0,16 \\
\hline LASct (\%) & $-0,31$ & 0,19 & $-0,08$ & 0,59 & $-0,01$ & 0,95 \\
\hline $\operatorname{LV}$ e'SR $^{\prime}\left(\mathrm{seg}^{-1}\right)$ & 0,15 & 0,54 & $-0,46$ & 0,05 & 0,17 & 0,49 \\
\hline Stiffness index & $-0,40$ & 0,09 & 0,24 & 0,33 & $-0,08$ & 0,76 \\
\hline
\end{tabular}

\section{Intra and interobserver correlation}

Good intra and interobserver correlation was observed. The results are detailed in Table 5.

Table 5

Intra and Interobserver Correlation

\begin{tabular}{|lllll|}
\hline & Intraobservador & \multicolumn{3}{l|}{ Interobservador } \\
\hline & IC 95\% & CCI & IC 95\% & CCI \\
\hline LASr & 0,98 & $0,92-0,99$ & 0,96 & $0,86-0,99$ \\
\hline LASRr & 0,95 & $0,82-0,98$ & 0,85 & $0,72-0,96$ \\
\hline LAScd & 0,97 & $0,93-0,99$ & 0,96 & $0,86-0,99$ \\
\hline LASRcd & 0,98 & $0,97-0,99$ & 0,98 & $0,95-0,99$ \\
\hline LASct & 0,95 & $0,83-0,98$ & 0,88 & $0,70-0,92$ \\
\hline LASRct & 0,96 & $0,85-0,98$ & 0,92 & $0,70-0,98$ \\
\hline $\begin{array}{l}\text { LASr: strain during reservoir phase; LASRr: peak strain rate during reservoir phase; LAScd: strain } \\
\text { during the conduit phase; LASRcd: peak strain rate during conduit phase; LASct: strain during } \\
\text { contraction phase; LASRct: peak strain rate during contraction phase. }\end{array}$ \\
\hline
\end{tabular}

\section{Discussion}

In the present study we have analyzed the LA function in a group of patients with a recent diagnosis of $\mathrm{HH}$ who have not received any specific treatment for this disease. They had no cardiovascular symptoms and Fe overload was documented by biochemical methods, MRI and liver biopsy. Despite not observing differences in LA size and volumetric parameters, we have found a significant decrease in longitudinal 
strain during the reservoir and conduit phases, with an increase in the stiffness index, with which we can infer an incipient compromise of atrial myocardial function.

The indexed LA volume is more robust as a predictor of events than the anteroposterior diameter or the LA area. Current guidelines propose its measurement, and a value greater than $34 \mathrm{~mL} / \mathrm{m}^{2}$ is associated with higher mortality, heart failure, atrial fibrillation, and ischemic stroke. Its value also correlates with other markers of ventricular diastolic dysfunction such as the E/e' ratio, and represents the impact over time of the increase in LV filling pressures (9). However, various techniques for studying myocardial function that could prove to be more sensitive have been developed. The study of myocardial deformation carried out using the speckle tracking technique has shown high feasibility and low interobserver variability. It has been increasingly used in the evaluation of myocardial function, mainly of the left ventricle, and in recent years its use has spread to the right ventricle, and to both atria. The method is based on monitoring the acoustic markers (speckles) of the myocardium during the cardiac cycle. It has the advantage of being angle independent, and the limitation of requiring adequate acoustic windows, which are not always possible. Its analysis has shown prognostic value in numerous clinical scenarios: coronary disease, heart failure, valvular disease, cardiomyopathy, and cardio oncology, among others $(5,13)$.

In the presence of Fe overload, this metal enters the myocyte through L-type calcium channels. The left ventricle is the most compromised heart chamber, and then in decreasing order are the right ventricle, the left and the right atrium (14). Intracellular Fe is found as ferritin, hemosiderin, and free form. The latter represents the most active form, which leads to the formation of toxic free radicals through the Fenton reaction. When the antioxidant capacity is exceeded, intracellular alterations occur at the level of the plasma membrane, proteins and nucleic acids that result in various degrees of dysfunction. On the other hand, there are alterations in the entry of calcium into the cell, which also contributes to alterations in systolic and diastolic function. It has been postulated that diastolic dysfunction is usually the earliest affectation of myocardial function in this pathology (8). In the LV, the subepicardial myocardium is mainly involved, so it is expected that in the analysis of contractile mechanics, radial and circumferential strain will be initially affected and therefore cardiac torsion, as observed by different studies, including ours (15-18).

In secondary hemochromatosis, Fe overload levels are usually much higher, and clinical cardiac involvement is also more frequent, but a significant correlation between serum ferritin levels and myocardial Fe concentration or the presence of cardiomyopathy has not always been observed, which makes us suppose that there are other immunoinflammatory and/or genetic mechanisms involved. Different studies have shown an early compromise of diastolic function in young patients with beta thalassemia and marked Fe overload (ferritin $>2000 \mathrm{ng} / \mathrm{mL}$ ). Kostopoulo et al observed an increase in LA size and a decrease in the active and passive ejection fraction of the left atrium compared with a control population (19), and in the same sense, a decrease in atrial strain has been observed during the reservoir phase (20). The compromise of the LA function could occur both as a consequence of the increase in its 
afterload due to the increased LV rigidity, as well as its intrinsic compromise by Fe deposits in the atrial myocardium.

In $\mathrm{HH}$, the clinical impact from the cardiovascular point of view is limited, when compared to forms secondary to repeated blood transfusions, where the Fe overload is much higher. However, subclinical compromise has been observed in both diastolic and systolic function in these patients, especially when more sensitive study techniques such as tissue Doppler or two-dimensional strain are used. Shizukuda $Y$ et al studied 43 patients with $\mathrm{HH}$, of whom half were recently diagnosed, and the others were under treatment by phlebotomies. Both groups were asymptomatic and were compared with a control population. No differences were observed in diastolic and systolic function between both groups by Doppler evaluation. However, patients with $\mathrm{HH}$ presented greater LA contraction force, which was assumed to be an early subclinical alteration of diastolic function in this group. There was no correlation with the serum concentrations of the Fe parameters, nor were there significant differences between the $\mathrm{HH}$ groups that had or did not have treatment for the disease (21). More recently, Byrne et al evaluated 25 patients with a recent diagnosis of $\mathrm{HH}$ and evaluated systolic function by analyzing $\mathrm{LV}$ radial strain and diastolic function through LA contraction force. After one year of treatment with phlebotomies, they observed a significant increase in radial strain $(36.4 \pm 21.3 \%$ vs $52.7 \pm 18.9 \%, p=0.015)$ and an improvement in diastolic function (5.6 kdynes vs 4.4 kdynes, $p=0.001)$. Unlike the previous work, in this case a difference was observed in cardiac function with the treatment, perhaps due to a longer time between both analyzes, and to the fact that patients were compared with themselves (4).

In our work we have found a moderate correlation between the LASr and the LV e'SR. Some research suggests that the E/ e'SR ratio could be a superior parameter to the classic tissue Doppler E/e' ratio in terms of prognostic value and a better correlation with LV filling pressures. Therefore, we could infer that the e' wave measured by strain rate could behave as an even more sensitive marker of diastolic alteration (22).

Other interesting information is the higher stiffness index in $\mathrm{HH}$ patients. It is defined as the pressure required to achieve a determined increase in volume in the LA (12). This easily obtained parameter has been postulated as an earlier marker of atrial function compromise and diastolic dysfunction, as well as being associated with follow-up events such as the appearance of atrial fibrillation and the occurrence of embolisms. It has been shown that the alteration of this index is even earlier than the increase in atrial volume, classically used as a prognostic variable (23-25). In a recent study by Porpáczy et al in a patient with systemic sclerosis, stiffness index is the variable that best correlates with serum levels of NT-proBNP, surpassing isolated LASr and LA volume (26).

We have not observed a correlation between various LA function parameters and the Fe metabolism profile. But the body excess of this metal, by the previously described mechanisms, generates reactive oxygen species, which would be the main determinants of cell dysfunction and death. It has been shown that patients with $\mathrm{HH}$ have elevated blood markers of oxidative stress, regardless of whether or not they are undergoing phlebotomy treatment, and that these correlate significantly with diastolic tissue 
velocities. Shizukuda et al observed a significant relationship between the velocities of the tissue Doppler e' wave and the levels of erythrocyte glutathione and malondialdehyde (27).

Although cardiac magnetic resonance imaging did not show pathological values of Fe overload, cardiac involvement cannot be ruled out. A recent study using the T1 mapping technique shows that up to two thirds of patients with systemic iron overload, with T2* greater than $20 \mathrm{~ms}$, could have myocardial deposits of this metal, which is why some researchers are already proposing T1 mapping as a complement to $\mathrm{T}^{*}$ in the evaluation of these patients (28).

\section{Limitations}

Among the limitations of our work are the lack of measurement of serum oxidative stress parameters and markers of myocardial dysfunction such as natriuretic peptides. However, in another research carried out by members of our team, not yet published, a significant decrease in serum levels of coenzyme Q10 (a powerful endogenous antioxidant) has been shown in patients with $\mathrm{HH}$.

\section{Conclusion}

Finally, we estimate that the present work provides relevant information to infer that in the early stages of $\mathrm{HH}$, there are early and subclinical alterations of LA function. It would be interesting to observe the behavior of these parameters in the follow-up, once the treatment for Fe overload has been started.

\section{Declarations}

\section{Conflict of interest:}

The authors declare that they have no conflict of interest.

\section{Funding:}

This study was not funded by any institution.

\section{References}

1. Brissot P, Pietrangelo A, Adams $P$ et al (2018) Haemochromatosis. Nat Rev Dis Primers. https://doi.org/10.1038/nrdp.2018.16

2. Muhlestein JB. Cardiac abnormalities in hemochromatosis. In: Eds Barton JC, Edwards CQ editors. Hemochromatosis. Genetics, pathophysiology, Diagnosis and Treatment. Cambridge University Press; 2011.p. 297-311 
3. Rozwadowska K, Daniłowicz-Szymanowicz L, Fijałkowski M, Sikorska K, Gałaska R, Kozłowski D et al (2018) Can two- dimensional speckle tracking echocardiography be useful for left ventricular assessment in the early stages of hereditary haemochromatosis? Echocardiography 35:1772-1781

4. Byrne D, Walsh JP, Daly C, McKiernan S, Norris S, Murphy RT et al (2020) Improvements in cardiac function detected using echocardiography in patients with hereditary haemochromatosis. Ir J Med Sci 189:109-117

5. Donal E, Behagel A, Feneon D (2015) Value of left atrial strain: a highly promising field of investigation. Eur Heart J Cardiovasc Imaging 16:356-357

6. Badano L, Kolias TJ, Muraru D et al (2018) Standardization of left atrial, right ventricular, and right atrial deformation imaging using two dimensional speckle tracking echocardiography: a consensus document of the EACVI/ASE/Industry TaskForce to standardize deformation imaging. Eur Heart J Cardiovasc Imaging 19:591-600

7. Bacon BR, Adams PC, Kowdley KV, Powell LW, Tavill AS, American Association for the Study of Liver Diseases (2011) Diagnosis and management of hemochromatosis: 2011 practice guideline by the American Association for the Study of Liver Diseases. Hepatology 54:328-343

8. Kremastinos DT, Farmakis D (2011) Iron overload cardiomyopathy in clinical practice. Circulation $124: 2253-2263$

9. Lang RM, Badano LP, Mor-Avi V et al (2015) Recommendations for Cardiac Chamber Quantification by Echocardiography in Adults: An Update from the American Society of Echocardiography and the European Association of Cardiovascular Imaging. J Am Soc Echocardiogr 28:1-39

10. Hoit BD (2014) Left atrial size and function: role in prognosis. J Am Coll Cardiol 63:493-505

11. Appleton CP, Kovács SJ (2009) The role of left atrial function in diastolic heart failure. Circ Cardiovasc Imaging 2:6-9

12. Kurt M, Wang J, Torre-Amione G, Nagueh SF (2009) Left atrial function in diastolic heart failure. Circ Cardiovasc Imaging 2:10-15

13. Cameli M, Mandoli GE, Loiacono F et al (2016) Left atrial strain: a new parameter for assessment of left ventricular filling pressure. Heart Fail Rev 21:65-76

14. Carpenter JP, He T, Anderson LJ et al (2011) On T2* Magnetic Resonance and Cardiac Iron. Circulation 123:1519-1528

15. Aladio JM, Yamasato F, Saad AK et al (2019) Two-dimensional Strain Echocardiography in the Evaluation of Ventricular Function in Patients with Newly Diagnosed Hereditary Hemochromatosis. Rev Argent Cardiol 87:449-457

16. Di Odoardo L, Giuditta M, Cassinerio E et al (2017) Myocardial deformation in iron overload cardiomyopathy: speckle tracking imaging in a beta-thalassemia major population. Intern Emerg Med 6:799-809

17. Monte I, Buccheri S, Bottari V et al (2012) Left Ventricular Rotational Dynamics in BetaThalassemia Major: A Speckle-Tracking Echocardiographic Study. J Am Soc Echocardiogr 25:1083-1090 
18. Rozwadowska K, Danilowicz-Szymanowicz L, Fijalkowski M et al (2018) Can two-dimensional speckle tracking echocardiography be useful for left ventricular assessment in early stages of hereditary haemochromatosis. Echocardiography 35:1772-1781

19. Kostopoulo A, Tsiapras DP, Chaidaroglou AS et al (2014) The pathophysiological relationship and clinical significance of left atrial function and left ventricular diastolic dysfunction in b-thalassemia major. Am J Hematol 89:13-18

20. Karamaou AG, Hamodraka ES, Vrakas SC et al (2014) Assessment of left ventricular and atrial diastolic function using two-dimensional (2D) strain imaging in patients with b-thalassemia major. Eur J Haematol 92:59-65

21. Shizukuda Y, Bolan CD, Tripodi DJ et al (2006) Significance of Left Atrial Contractile Function in Asymptomatic Subjects With Hereditary Hemochromatosis. Am J Cardiol 98:954-959

22. Højbjerg Lassen MS, Olsen FJ, Skaarup KG et al (2020) The clinical application of the ratio of transmitral early filling velocity to early diastolic strain rate: a systematic review and meta-analysis. J Echocardiogr 18:98-104

23. Deschle HA, Gantesti J, Culaciati G et al (2014) Left atrial longitudinal strain: Early alterations in Young Patients with Mild Hypertension. Rev Argent Cardiol 82:126-132

24. Demir M, Aktaş, Yıldırım A (2017) Left atrial mechanical function and stiffness in patients with non dipper hypertension: A speckle tracking study. Clin Exp Hypertens 39:319-324

25. Pilichowska-Paszkiet E, Baran J, Sygitowicz G et al (2018) Noninvasive assessment of left atrial fibrosis. Correlation between echocardiography, biomarkers, and electroanatomical mapping. Echocardiography 35:1326-1334

26. Porpáczy A, Nógrádi A, Vértes V et al (2019) Left atrial stiffness is superior to volume and strain parameters in predicting elevated NT-proBNP levels in systemic sclerosis patients. Int $\mathrm{J}$ Cardiovasc Imaging 10:1795-1802

27. Shizukuda Y, Bolan CD, Tripodi DJ et al (2009) Does Oxidative Stress Modulate Left Ventricular Diastolic Function in Asymptomatic Subjects with Hereditary Hemochromatosis? Echocardiography 26:1153-1158

28. Torlasco C, Cassinerio E, Roghi A et al (2018) Role of T1 mapping as a complementary tool to T2*for non- invasive cardiac iron overload assessment. PLoS One 13:e0192890

\section{Figures}




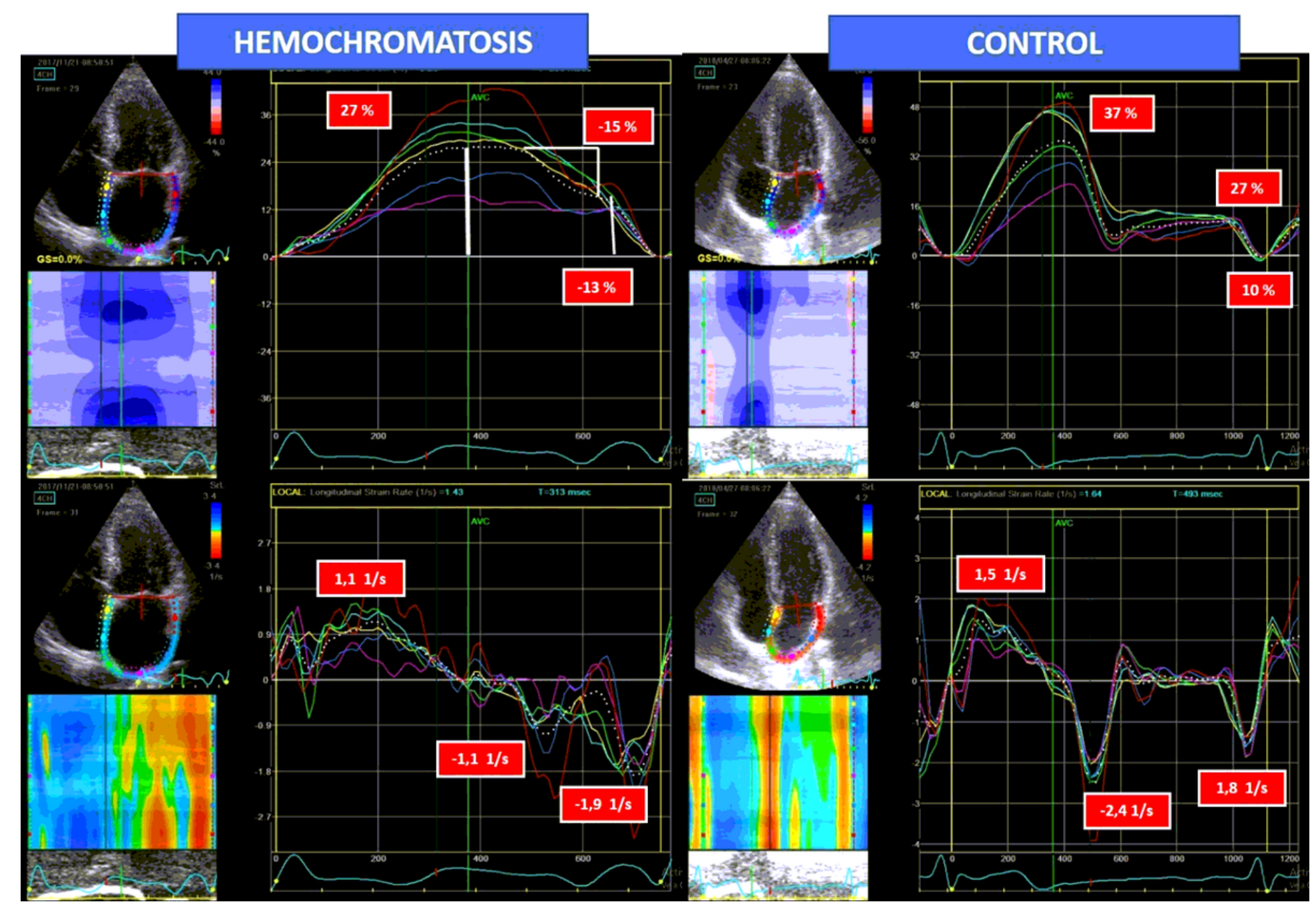

Figure 1

Left atrial strain curves of a patient with $\mathrm{HH}$ and a control one. 


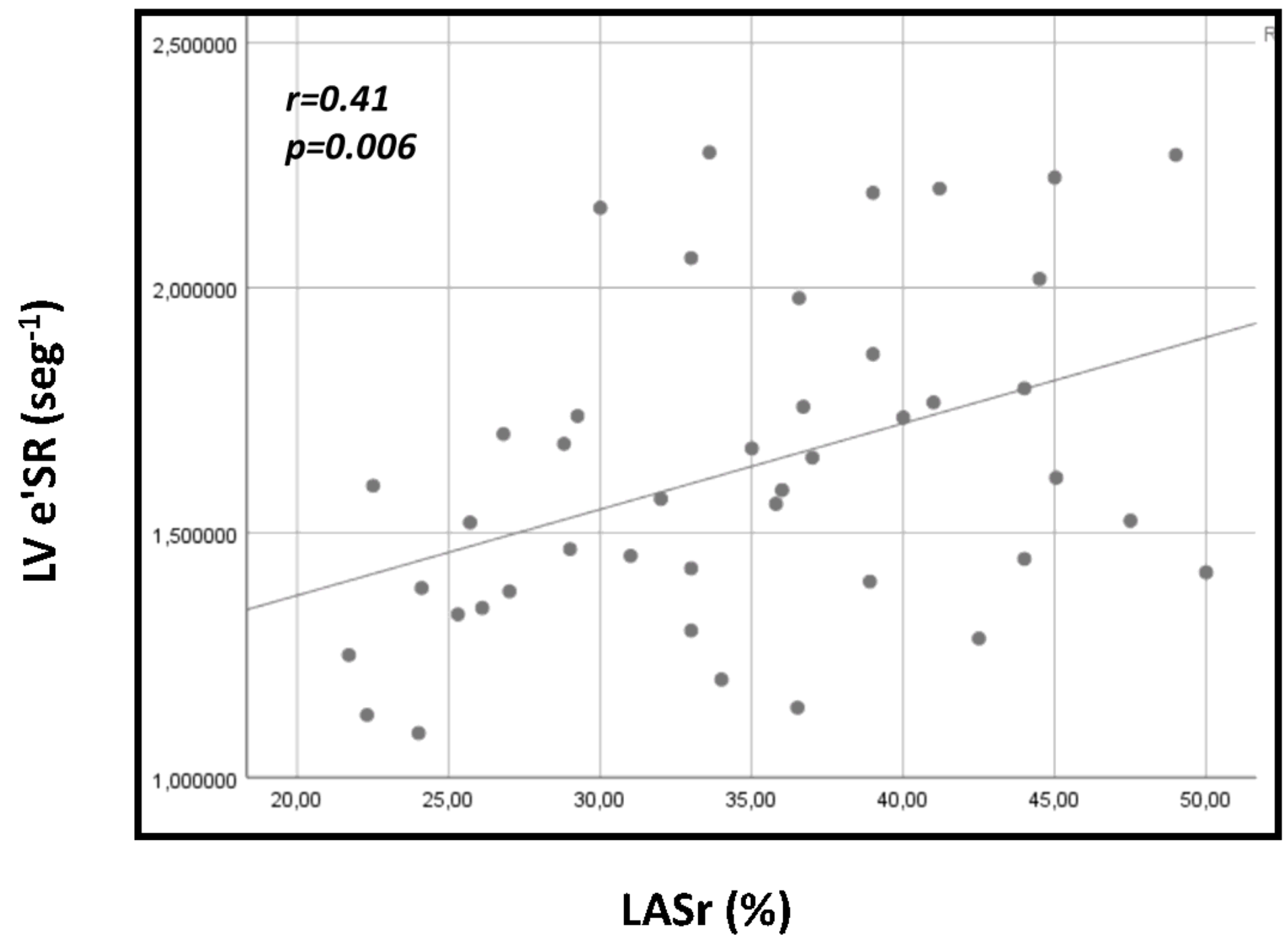

Figure 2

Correlation between the LA strain during reservoir phase and the LV e'SR. 